Ebisu

Études japonaises

Études japonaises

$54 \mid 2017$

L'après-guerre des intellectuels japonais

\title{
Le différend : la question des responsabilités de guerre et de la réorientation chez Yoshimoto Takaaki entre 1955 et 1958
}

吉本隆明における戦争責任と転向の問題一1955年から1958年にかけて

The Dispute: War Responsibilities and Ideological Conversion in Yoshimoto

Takaaki's Work between 1955 and 1958

Éric Dumont et Vincent Manigot

\section{OpenEdition}

Journals

Édition électronique

URL : http://journals.openedition.org/ebisu/2058

DOI : 10.4000/ebisu.2058

ISSN : 2189-1893

Éditeur :

Institut français de recherche sur le Japon (UMIFRE 19 MAEE-CNRS), Maison franco-japonaise

Édition imprimée

Date de publication : 19 décembre 2017

Pagination : 113-146

ISSN : 1340-3656

\section{Référence électronique}

Éric Dumont et Vincent Manigot, «Le différend : la question des responsabilités de guerre et de la réorientation chez Yoshimoto Takaaki entre 1955 et 1958 », Ebisu [En ligne], 54 | 2017, mis en ligne le 19 décembre 2017, consulté le 01 mai 2019. URL : http://journals.openedition.org/ebisu/2058 ; DOI : 10.4000/ebisu. 2058 


\section{Le différend}

La question des responsabilités de guerre et de la réorientation chez Yoshimoto Takaaki entre 1955 et 1958

\section{Éric Dumont \& Vincent Manigot}

吉本隆明におけろ戦争責任と転向の問題

1955 年から 1958 年にかけて

エリック・ジュモン、ヴァンサン・マニゴ

The Dispute: War Responsibilities and Ideological Conversion in Yoshimoto Takaaki’s Work between 1955 and 1958

Éric Dumont \& Vincent Manigot

Mots-clés : Après-guerre, communisme, littérature, pensée, responsabilité de guerre, Seconde Guerre mondiale, tenkō, Yoshimoto Takaaki.

L'auteur : Éric Dumont est doctorant à l'université de Tokyo. Ses recherches portent principalement sur le théâtre moderne et contemporain, ainsi que sur la littérature et la pensée du Japon.

Vincent Manigot est doctorant à l'Inalco. Ses recherches portent principalement sur l'histoire de l'art moderne et contemporain et le surréalisme japonais.
Résumé : La carrière de penseur critique $\mathrm{du}$ poète Yoshimoto Takaaki prend son essor au cours des années 1950, à travers notamment un texte remarqué sur la réorientation dans lequel il établit une équivalence entre réorientés et non-réorientés. Nous montrons que sa réflexion sur la réorientation s'inscrit dans le prolongement d'un travail polémique mené avec Takei Teruo sur la responsabilité de guerre des écrivains progressistes. En présentant le développement, le contexte intellectuel, certains protagonistes ainsi que les enjeux de cette polémique, nous tentons de cerner l'originalité de la démarche de Yoshimoto. 
】キーワード

戦後、共産主義、文学、思想、戦争責任、 第二次世界大戦、転向、吉本隆明

\section{著者}

エリック・ジュモンは東京大学博士課程在学。

日本の近現代演劇を中心に、文学や思想につ いて研究している。

ヴァンサン・マニゴはフランス国立言語文化 大学博士課程在学。日本の近現代美術史と シュルレアリスムについて研究している。
要旨

詩人吉本隆明は 1950 年代半ば、とりわけ非転 向者と転向者の類似性を主張し注目を浴びた エッセイ『転向論』により、思想家として頭角を 現す。彼の転向論は、進歩的文学者の戦争責 任についての武井昭夫との共同批評活動の延長 として繰り広げられているだうう。この論争につ いて、その展開や思想的文脈、関係する当事 者たち、また問題点について論じつつ、吉本の アプローチの独自性を明らかにしたい。
Veywords: Postwar, Communism, literature, thought, war responsibility, Second World War, ideological conversion, Yoshimoto Takaaki.

The Author: Éric Dumont is a Ph.D. candidate at the University of Tokyo. His research focuses on modern and contemporary Japanese theatre, as well as Japanese literature and philosophy.

Vincent Manigot is a Ph.D. candidate at Inalco. His research focuses on Japanese modern and contemporary art, and the reception of Surrealism in Japan.

\begin{abstract}
The poet Yoshimoto Takaaki's career as a critical thinker took off in the 1950 s, notably through a widely remarked essay on ideological conversion in which he established an equivalence between converts and non-converts. We argue that his thoughts on ideological conversion were rooted in the polemic work he engaged in - along with Takei Teruo - on the war responsibility of progressive writers. We seek to identify the originality of Yoshimoto's approach by discussing and presenting the development of this controversy as well as its actors, implications and general intellectual context.
\end{abstract}




\section{Le différend}

\section{La question des responsabilités de guerre et de la réorientation chez Yoshimoto Takaaki entre 1955 et 1958}

Éric DUMONT et Vincent MANIGOT*

\section{Retour sur Yoshimoto Takaaki}

Dans le paysage de la pensée contemporaine japonaise, Yoshimoto Takaaki 吉本隆明 (1924-2012) occupe à plusieurs égards une place à part. Figure charismatique de l'agitation des années 1960, il serait devenu, après l'éclatement de la bulle, un intellectuel obscur et conservateur. Précurseur des études postcoloniales pour certains ${ }^{1}$, il aurait été, pour d'autres, éclipsé par

1. Murakami Fuminobu considère que le travail de Yoshimoto anticipe sur certains points celui de Gayatri Spivak, dont les travaux ont contribué à former le champ des études subalternes (Murakami 2005 : 95-135).

* Université de Tokyo et INALCO, respectivement. 
l'émergence du poststructuralisme $e^{2}$. De nos jours, l'appréciation de son œuvre au Japon apparaît pour le moins contrastée ${ }^{3}$, comme si le dédain contemporain cherchait à répondre à la fascination d'antan. À ce titre, rares sont, au Japon, les intellectuels dont la réputation polarise autant les avis que celle de Yoshimoto ${ }^{4}$.

Cette méfiance vis-à-vis de ses travaux, ou de leurs apports éventuels, ne rime pas pour autant avec un désintérêt pour la figure de Yoshimoto. Les approches récentes les plus intéressantes à nos yeux, celles d'Oguma Eiji et de Suga Hidemi notamment, le considèrent ainsi comme phénomène ou symptôme d'une époque, et focalisent leurs analyses critiques sur les liens historiques unissant Yoshimoto à son milieu ${ }^{5}$. Elles s'attachent à

2. C'est du moins ce que laisse entendre Karatani Kōjin lorsqu'il affirme que Yoshimoto s'efforçait d'exprimer, dans un langage inadéquat, ce que le poststructuralisme considéra par la suite comme l'évidence même, dans une langue appropriée (Karatani 1996 [1982] : 244-257).

3. En dehors du Japon, l'œuvre de Yoshimoto reste peu connue. Les travaux qui lui sont consacrés, comme les traductions, sont rares. À l'exception de quelques poèmes dans des anthologies, et d'un court texte publié dans le catalogue de l'exposition « Le Japon des avant-gardes, 1910-1970 » (Yoshimoto 1986b : 230-236), il existe à notre connaissance trois essais traduits dans leur intégralité : «Les fondements intellectuels de l'autonomie » (Jiritsu no shisōteki kyoten 自立の思想的拠点) (Yoshimoto 1996 [1964] : 186-224), “ On tenkō, or ideological conversion» (Tenkō-ron 転向論) (Yoshimoto 2011 [1958] : 103-121), Karl Marx (traduction commentée dans le cadre de la thèse de doctorat de Manuel Yang, Yoshimoto Taka'akis Karl Marx: Translation and Commentary, University of Toledo, 2008, 249 p.), et un entretien avec Michel Foucault « Méthodologie pour la connaissance du monde : comment se débarrasser du marxisme " (in Dits et écrits, 19541988, vol. 3, Paris, Gallimard, 1994, p. 595-618). On trouvera par ailleurs une présentation très complète de Yoshimoto, accompagnée de résumés de certains de ses grands travaux dans Modern Japanese Culture de Leith Morton (Morton 2003 : 103-155).

4. Quoique abusive sur plusieurs aspects, la comparaison qu'établit Suga Hidemi avec la figure de Jean-Paul Sartre nous paraît instructive sur certains points. Il est en effet probable que leur appartenance au monde des Lettres, ainsi que leur extériorité par rapport à l'Université, aient joué un rôle favorable au moment du lancement de leurs carrières respectives, et que ces mêmes éléments se soient par la suite retournés contre eux (Suga $2008: 26-30$ ).

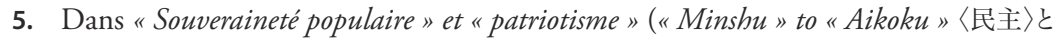
〈愛国〉, Oguma Eiji consacre plusieurs analyses, ainsi qu'un chapitre entier à Yoshimoto. Il considère que l'ascension de Yoshimoto est le résultat de l'exacerbation, au milieu des années 1950, d'un conflit générationnel opposant ceux qui sont nés avant 
déconstruire les divers mythes forgés autour du personnage, et tentent de resituer son œuvre dans le climat d'après-guerre.

C'est en effet au cours des années 1950 que sa carrière de penseur critique prit son essor, à travers un réexamen polémique de la responsabilité de guerre des écrivains. Nous nous intéresserons à cette polémique, dont nous tenterons de présenter le contexte, retracer le développement et déterminer certains enjeux. L'un de ces enjeux se situe autour d'une redéfinition de la notion de réorientation $(t e n k \bar{o} \text { 転向 })^{6}$. Cette dernière est en effet très présente dans les essais critiques de Yoshimoto entre 1954 et 1958. Nous nous attacherons à suivre et analyser les variations et évolutions du concept de réorientation dans sa pensée, en les confrontant à certaines approches qui lui sont contemporaines, afin d'en saisir les spécificités.

\section{La responsabilité de guerre des écrivains}

À l'heure où une coalition politique fraîchement formée, le Parti Libéral Démocrate (Jiyūminshutō 自由民主党), claironnait après son arrivée au pouvoir que l'après-guerre appartenait désormais au passé, deux critiques littéraires alors inconnus du grand public, Yoshimoto Takaaki et Takei

la guerre (senzenha 戦前派) à ceux qui sont nés pendant (senchūha 戦中派), que leurs expériences respectives du conflit, et surtout de la défaite, sépareraient (Oguma 2002: 598-655). Suga, quant à lui, insiste sur une certaine fiction stratégique, ou mise en scène de soi, que Yoshimoto développa au cours des nombreuses polémiques qui jalonnent son œuvre.

6. Si en anglais, l'usage du syntagme ideological conversion semble tant bien que mal s'imposer, notons qu'il n'existe en français pour le moment pas d'expression consacrée pour traduire ce terme (voir Fauconnier 2012 : 10-15 pour une présentation générale de la notion, 55-56 pour une recension non-exhaustive des traductions françaises). Soulignons par ailleurs qu'il n'est pas rare de rencontrer dans un même texte plusieurs " équivalents " différents (tel est notamment le cas pour la traduction de "On tenkō" par Wake Hisaaki [Yoshimoto 2011]). Si la diversité apporte parfois quelque louable précision, il nous semble que cette prolifération de synonymes risque en définitive de nuire à la cohérence d'une notion sensible et difficile à saisir. Nous privilégierons donc ici, malgré les limitations qu'impose l'exercice, la stabilité lexicale et adopterons pour ses aspects fonctionnels d'une part et sa proximité avec le japonais d'autre part, la traduction « réorientation ". 
Teruo 武井昭夫 (1927-2010), rallumaient les braises d'un débat vieux d'une dizaine d'années : celui des responsabilités de guerre. Si tous deux appartenaient au monde des Lettres, leurs parcours ne s'étaient encore jamais croisés. À la différence de Yoshimoto, qui avait vécu en marge de la politique, Takei était, lui, un activiste chevronné. Il avait rejoint le Parti communiste japonais (Nihon kyōsantō 日本共産党, ci-après PCJ) en 1946, puis avait milité pour la création de la Fédération japonaise des associations d'autogestion étudiantes (Zen-Nihon gakusei jichikai sōrengō 全日本 学生自治会総連合) dont il devint en 1948 le premier Premier secrétaire. Il intégra en 1947 la Société Littéraire du Nouveau Japon (Shin-Nihon bungaku-kai 新日本文学会, ci-après SLNJ), mais n'y devint actif qu’à partir du début des années 1950. Si les circonstances exactes de leur rencontre restent incertaines ${ }^{7}$, les fruits qu'elle porta sont, eux, connus : un recueil cosigné qui devait susciter le débat, La responsabilité de guerre des écrivains (Bungakusha no sensō sekinin 文学者の戦争責任).

L'ouvrage, publié à l'automne 1956 par les éditions Awaji shobō 淡路 書房, reprend en réalité une série d'articles que Yoshimoto et Takei avaient d'abord fait paraître à titre individuel dans diverses revues ${ }^{8}$, et dans lesquels

7. Lors d'une interview organisée par Karatani Kōjin, pour le vingtième numéro de la revue Espace critique (Hihyö kükan 批評空間, janvier 1999), Takei mentionne l'existence d'un cercle d'études formé en juillet 1955, réunissant Yoshimoto, le critique littéraire Okuno Takeo 奥野健男 (1926-1997), le romancier Hino Keizō 日野啓三 (1929-2002) et lui-même. Sachant que ces mêmes personnes sont à l'origine de la revue Critique contemporaine (Gendai hyōron 現代評論), dont le premier numéro, en décembre 1958, contient «La réorientation » (Tenkō-ron 転向論) de Yoshimoto, nous pouvons imaginer qu’il existait sans doute une certaine cohésion au sein du groupe (Takei 2004 [1999] : 131).

8. Outre une préface et une postface rédigées pour l'occasion, La responsabilité reprend les articles suivants: Yoshimoto Takaaki, "Notes sur Takamura Kōtarō " (Takamura Kōtarō nōto 高村光太郎ノート) (Gendai-shi 現代詩 [Poésie contemporaine], juillet 1955, il ne s'agit ici que de la partie concernant la période de guerre), "Les poètes de la génération précédente " (Zensedai no shijintachi 前世代の詩人たち) (Shigaku 詩学 [Poétique], novembre 1955), "Critique de la "littérature démocratique" " (Minshushugi bun$g a k u$ »hihan「民主主義文学」批判) (Arechi shishü荒地詩集 [Recueil de poèmes du groupe Arechi], avril 1956), "Les poètes d'après-guerre » (Sengo shijin-ron 戦後詩人論) (Shigaku 詩学, juillet 1956) ; Takei Teruo : «La responsabilité de guerre d'après-guerre et la littérature démocratique" (Sengo no sensō sekinin to minshushugi bungaku 戦後の戦争 責任と民主主義文学) (Gendai-shi, mars 1956), «Avant-garde politique et avant-garde 
ils interrogeaient, de manière encore inédite, la responsabilité de guerre des écrivains. Au sortir de la guerre, la SLNJ s'était la première emparée du problème ; aussi, dans la mesure où Yoshimoto et Takei lui succèdent, leurs travaux sont parfois qualifiés de « deuxième polémique sur la responsabilité de guerre des écrivains ». Cette appellation est toutefois quelque peu trompeuse car, plus qu'un prolongement, il s'agit en fait d'une reformulation.

La particularité de leur démarche consiste par ailleurs à considérer la question des responsabilités de guerre en relation avec ce qu'ils nomment la « responsabilité d'après-guerre " (sengo sekinin 戦後責任). Leurs travaux s'inscrivent, en ce sens, dans un contexte plus large, qui voit émerger alors, sur plusieurs fronts, une critique gauchisante du PCJ et de ses cadres ${ }^{9}$. Pour des raisons qui leur sont propres, Takei et Yoshimoto nourrissent chacun de profonds doutes au sujet du bien-fondé des thèses soutenues par le parti et de son fonctionnement ${ }^{10}$. L'histoire du PCJ dans l'après-guerre, de son revirement inexpliqué pendant la grève générale de février 1947, aux échauffourées du "premier mai sanglant " de 1952, sans compter les nombreuses querelles intestines, parfois meurtrières, menées au nom de l'unité, est en

artistique" (Seiji no avangyarudo to geijutsu no avangyarudo 政治のアヴアンギャルドと 芸術のアヴアンギャルド) (Bijutsu hihyō 美術批評 [Critique d'art], mars 1956), «D’un vulgaire scarabée sacré à un scarabée sacré révolutionnaire " (Zokuryū sukarabē sakure kara kakumeiteki sukarabē sakure e 俗流スカラベーサクレから革命的スカラベーサクレへ) (Bijutsu hihyō, mai 1956) et "Littérature d'après-guerre et avant-garde " parties une et deux (Sengo bungaku to avangyarudo 戦後文学とアヴアンギャルド) (Bijutsu hihyō, août et septembre 1956). Précisons d'une part qu'il s'agit du seul ouvrage cosigné par Yoshimoto et Takei, et d'autre part que ces articles furent par la suite repris, parfois remaniés voire enrichis, dans des recueils publiés à titre individuel. De même que pour Yoshimoto, toutes les citations de Takei proviennent de l'édition la plus récente.

9. Pour une synthèse de ces critiques, voir Suga 2008 : 121-142.

10. Sans trop entrer dans les détails, mentionnons que les activités syndicales de Yoshimoto lui coûtèrent plusieurs postes. Déjà méfiant du soutien que le PCJ était capable d'apporter aux grévistes, il vit d'un très mauvais œil le renoncement à la lutte armée décrété lors du sixième congrès du parti (27-29 juillet 1955) (voir Oguma 2002 : 626-627). Quant à Takei, il vécut le déchirement du PCJ et les luttes de pouvoir entre factions régionaliste (shokan-ha 所感派) et internationaliste (kokusai-ha 国際派) de l'intérieur du parti d'une part, de la SLNJ d'autre part. Il avait en outre développé, du fait de son activisme à l'université, plusieurs théories sur le rôle des étudiants au sein du mouvement social, que les cadres du parti avaient condamnées (Takei 2004 [1999]: 129-130). 
effet criblée de contradictions, dont aucun cadre ne fut amené à répondre. Il n'est dès lors pas impossible d'envisager que, pour Yoshimoto et Takei, le retour sur les responsabilités de guerre pouvait constituer un moyen détourné de critiquer cette situation.

Compte tenu du caractère gigogne de La responsabilité, il nous paraît primordial d'esquisser le contexte intellectuel de la relance de cette polémique en 1956, ainsi que d'exposer brièvement le contenu et les apories du premier débat, auquel elle entend répondre. Nous présenterons les procédés et arguments employés par Yoshimoto et Takei ${ }^{11}$, à travers lesquels nous tenterons d'analyser les usages et l'importance de la notion de réorientation.

\section{La SLNJ et le degré zéro de la responsabilité}

En mars 1956, l'historien des idées Maruyama Masao 丸山眞男 (19141996) remarque un récent regain d'intérêt pour la question des responsabilités de guerre des intellectuels (Maruyama 1995 [1956] : 159-164). Concédant que dans la configuration politique actuelle ce thème pourrait faire le jeu des forces réactionnaires, il affirme néanmoins que le mouvement

11. Nous ne chercherons donc pas à établir de distinction particulière entre les positions de Takei et Yoshimoto dans leur entreprise. Il est évident que leurs trajectoires respectives étaient, à l'époque déjà, aussi différentes qu'elles le seront par la suite. Alors que Yoshimoto se lancera à la recherche d'une alternative au marxisme en tentant de développer, notamment, une théorie de l'autonomie (voir Yoshimoto 1996 [1964] : 183-224, et Olson 1978 : 340-352), Takei, lui, poursuivra ses activités au PCJ comme à la SLNJ, dont il deviendra rédacteur en chef au cours des années 1960. Nous n'ignorons pas non plus qu'une violente querelle opposa les deux hommes au début des années 1960. Il ne nous semble toutefois pas inutile de nuancer la centralité qu'accordent certaines approches à la personnalité de Yoshimoto. La plus récente nous semble être celle d'Oguma Eiji, qui ne mentionne Takei qu'à deux reprises (Oguma 2002 : 287, 290), mais en dehors du chapitre consacré à Yoshimoto (Oguma 2002 : 598-655). Rien n’indique, en effet, que l'influence de Yoshimoto sur Takei ait été unilatérale. Il nous paraît également important d'éviter certains écueils qui, de manière rétrospective, anticipent les divergences qui les sépareront, comme le fait par exemple Soeda Kaoru (Soeda 2010 : 25-30). Rien n'annonce, pourtant, dans leurs articles de l'époque, leurs désaccords à venir, ni ne signale une différence fondamentale de point de vue sur la question. Nous pensons donc, comme le suggère Yuchi Asao que, sans les confondre, il faut toutefois postuler une "communauté d'idées " entre Yoshimoto et Takei dans La responsabilité (Yuchi $2006: 273$ ). 
pour la paix, comme celui pour la défense de la Constitution, ne pourront progresser en refusant d'y faire face. Le problème mérite toutefois, à ses yeux, d'être reformulé. Il ne doit pas se limiter d'une part aux seuls intellectuels, mais être au contraire étendu à tous les groupes sociaux. D'autre part, il lui faut admettre des formes de responsabilités qui échappent au registre juridique, notamment celle de la responsabilité politique. Or, sur ce dernier point, avance Maruyama, la question des responsabilités de guerre connaît deux angles morts : l'empereur d'un côté, le PCJ «ou plus précisément les communistes non-réorientés " (ibid. : 163) de l'autre. Si le problème de la non-responsabilité de l'empereur renvoie évidemment à la décision américaine de ne pas le faire comparaître lors du procès de Tokyo, interroger la responsabilité de guerre des "communistes non-réorientés " semble de prime abord plus surprenant. On constate que la thématique des responsabilités de guerre, non plus seulement celle des dirigeants jugée au lendemain des hostilités, mais celle plus diffuse et surtout bien moins saisissable des intellectuels et artistes japonais, est alors particulièrement interrogée. Au sujet des "non-réorientés ", Maruyama adopte une posture assez consensuelle, estimant qu' « il n'y a à douter ni du courage ni de l'intégrité dont ils firent preuve en endurant toutes les pressions et persécutions dans leur lutte contre la guerre et le fascisme» (ibid. : 164). Les discours leur conféraient en effet alors une place bien particulière quant à la question des responsabilités de guerre ${ }^{12}$.

Le terme de "non-réorienté " désigne une infime minorité d'individus, pour la plupart communistes, tels Tokuda Kyūichi 徳田球一 (18941953), Nosaka Sanzō 野坂参三 (1892-1993) ou Miyamoto Kenji 宮本 顕治 $(1908-2007)^{13}$. Leur refus de céder face à l'ennemi fit, dans l'immédiat

12. Ce passage s'appuie sur Oguma (2002: 175-190), Dower (1999: 233-244), et Koschmann (1993: 395-414).

13. Avocat de formation, Tokuda fut arrêté en 1928. Il devint, après dix-huit ans de prison, Premier secrétaire du PCJ en 1945. De retour d'Angleterre où il avait adhéré au Parti communiste en 1920, Nosaka participa en 1922 à la création du premier Parti communiste (illégal) au Japon. Lui aussi arrêté en mars 1928, il fut libéré pour raisons de santé et partit à l'étranger, en URSS notamment où il intégra le Komintern, puis en Chine, où il lutta aux côtés de la résistance "anti-japonaise ». Il rentra au Japon en 1945, et participa à la renaissance du PCJ, dont il fut Premier secrétaire de 1955 à 1958. Économiste de formation, Miyamoto s'était également distingué comme critique 
après-guerre, l'objet d'un respect quasiment unanime ${ }^{14}$. Au sein des milieux intellectuels progressistes, hantés par un double sentiment d'impuissance et de remord, ces "non-réorientés " pouvaient aussi incarner une certaine forme de constance ou de fidélité à des convictions. Par ailleurs, suite à sa reformation en décembre 1945, le PCJ embrassa dans un premier temps l'agenda des réformes entreprises par le $\mathrm{SCAP}^{15}$, et se présenta officiellement, tout en qualifiant les troupes américaines d'" armée de libération ", en champion de la "révolution démocratique " (Tokuda $1946: 3$ ). Si ce dernier point tenait sans doute surtout à l'attachement de ses cadres aux thèses de 1932, notamment l'idée de révolution en deux stades, cette position n'allait toutefois pas sans susciter quelques équivoques. D’une part, elle situait a priori le PCJ dans le camp des vainqueurs ${ }^{16}$. D'autre part, et malgré le concours du parti à l'avènement d'un «nouveau Japon », celuilà n'était-il pas plutôt en train de relancer un programme avorté dans les années 1930 ?

Ces questions ne seront toutefois ouvertement soulevées qu'après 1955. Soulignons pour le moment que, dans les ruines de la défaite et ce jusqu’à l'amorce du fameux « retour en arrière" (gyaku kōsu 逆コース), l'influence des communistes dans le champ intellectuel était considérable et la présence parmi eux de non-réorientés rendait leur légitimité pour ainsi dire indiscutable, encourageant en cela le retour de nombreux réorientés ${ }^{17}$.

Dans les milieux culturels, la tendance générale fut assez semblable. Des groupes se formèrent à nouveau, de nouveaux projets virent le jour. En décembre 1945, d'anciens membres de la Ligue japonaise des écrivains prolétariens (Nihon puroretaria sakka dōmei 日本プロレタリア作家同盟, ou NALP) se réunirent à Kanda pour l'inauguration de la SLNJ. Nous pouvons

littéraire avant son arrestation en 1933. Comme Tokuda, il ne fut libéré qu’en 1945. Il occupa, lui aussi, le poste de Premier secrétaire de 1958 à 1970.

14. Selon Oguma, MacArthur lui-même aurait considéré Tokuda et Nosaka, tout en déplorant le fait qu'ils soient communistes, comme des "types compétents " (yūnō na renchū 有能な連中) (Oguma $2002:$ 184).

15. Sur ce point, on consultera notamment Fauconnier (2007), Koschmann (1993: 397-401) et Lucken (2013 : 214-218).

16. C’est précisément la question soulevée par Maruyama (1995 [1956] : 164).

17. Le succès du PCJ au niveau électoral durant la seconde moitié des années 1940 participe de ce même élan (Fauconnier 2012 : 450). 
lire, dans le procès-verbal que la société publia dans le premier numéro de sa revue en mars 1946 : "Cette réunion n'est pas la simple renaissance des groupes prolétariens. Suivant les avancées de la révolution démocratique, nous visons le ralliement de tous les représentants de la littérature démocratique, et devons lutter pour son progrès" (Iwakami 1946 : 62).

Ce fut du reste sous cette appellation de « littérature démocratique » que la SLNJ entendait mener ses activités : produire une littérature à même de combattre les vestiges de l'ancien ordre semi-féodal, et lutter pour le renforcement de la révolution démocratique. Cet appel en faveur d'un "front commun " littéraire était probablement sincère, dans la mesure où l'invitation fut lancée bien au-delà du cercle des anciens de la NALP'18, et que la revue ouvrit par la suite régulièrement ses colonnes à des auteurs qui ne lui étaient pas affiliés ${ }^{19}$. La différence qu'invoquait la SLNJ entre son programme et celui des groupes prolétariens d'avant-guerre était en revanche plus trouble. En effet, le communiqué précisait encore : "Nous sommes parvenus à la conclusion que seuls ceux qui n'ont pas collaboré à cette guerre impérialiste et s'y sont opposés étaient qualifiés pour en être les fondateurs $»^{20}$ (Iwakami 1946: 62). Autrement dit, le critère qualificatif décisif consistait à avoir "les mains propres ", et sous-entendait implicitement une certaine forme de loyauté envers la NALP. La lutte décrétée contre les mouvements littéraires jugés réactionnaires ne tarda pas à s'engager. Au mois de juin, dans un article intitulé « Poursuite des responsabilités de guerre en littérature ", le critique littéraire Odagiri Hideo 小田切秀雄 (1916-2000) déclarait :

Les responsabilités de guerre en littérature sont avant tout, et plus que tout autre, notre propre problème. Ce problème commence par notre propre autocritique. Dans un monde libre, la supercherie ne prend pas. Ce que nous avons fait pendant

18. Pour quelques chiffres, voir Dower $(1999: 238)$.

19. Tel fut notamment le cas pour Yoshimoto qui entre 1954 et 1959 y publia une cinquantaine de billets, notes de lectures, critiques de films et autres petits essais.

20. La liste comprend les noms suivants : Akita Ujaku 秋田雨雀 (1883-1962), Eguchi Kan 江口渙 (1887-1975), Kubokawa Tsurujirō 洼川鶴次郎 (1903-1974), Tsuboi Shigeji 嗀井繫治 (1897-1975), Tokunaga Sunao 徳永直 (1899-1958), Nakano Shigeharu 中野 重治 (1902-1979), Fujimori Seikichi 藤森成吉 (1892-1977), Miyamoto Yuriko 宮本 百合子 (1899-1951) et Kurahara Korehito 蔵原惟人 (1902-1991). Tous avaient été impliqués dans la NALP. 
la guerre, c'est à nous de l'interroger, à nous de l'examiner, à nous de le critiquer. Nous comptons, ce faisant, tirer au clair nos propres responsabilités quant à l'état affligeant de décadence et de déchéance qu'a connu la littérature japonaise au cours de ces dix dernières années. (Odagiri 1946 : 64)

Odagiri avait par ailleurs pris soin dans son texte d'inclure une liste de vingt-cinq auteurs, sélectionnés, selon lui, sur la base de leur degré d'implication dans le conflit ou encore de leur renommée à l'intérieur et en dehors du monde littéraire ${ }^{21}$. Ces derniers étaient d'une part sommés de s'expliquer sur leur comportement, d'autre part de reconnaître leurs responsabilités et éventuellement de quitter les Lettres. Cette procédure entreprise par la SLNJ ne comptait donc pas étendre ses verdicts au-delà du monde littéraire, et assumait pleinement en cela sa nature extra-judiciaire. Le découpage qu'elle opérait entre ceux qui avaient activement apporté leur soutien au régime impérial et à la guerre, ceux qui ne les avaient soutenus que passivement et ceux qui leur avaient résisté, assignant ainsi un degré de responsabilité totale aux premiers, partielle aux seconds et nulle aux derniers, reflétait en revanche un système de représentations plus largement partagé, ou éventuellement tacitement admis, chez les intellectuels ${ }^{22}$. Cela ne signifie pas que les accusés s'exécutèrent, ou acceptèrent leur " peine ", mais que, sur le terrain de la responsabilité de guerre des écrivains, la démarche de la SLNJ ne connut pas de modèle véritablement concurrent, et conserva ainsi une certaine forme de mainmise sur la question jusqu'en 1956.

Néanmoins, pour paraphraser Maruyama, la formulation du problème par la SLNJ comportait quelques angles morts. Premièrement, la tripartition à laquelle elle se livrait était loin d'aller de soi. En effet, si aucun

21. Liste qui contient, entre autres, les noms de personnalités majeures de la scène culturelle de la première moitié du siècle, parmi lesquels l'écrivain et critique Kobayashi Hideo 小林秀雄 (1902-1983), le poète et sculpteur Takamura Kōtarō 高村光太郎 (18831956), ou encore deux importants représentants du romantisme japonais, l'écrivain et critique Kamei Katsuichirō 亀井勝一郎 (1907-1966) et le critique Yasuda Yojūrō 保田 與重郎 (1910-1981).

22. Karatani souligne à ce propos que l'approche de Maruyama, tout comme celle des membres de la revue Littérature moderne (Kindai bungaku 近代文学), butait sur le respect moral envers les non-réorientés dont l'un comme les autres ne parvenaient pas à se départir (Karatani 2003 [2000] :175-176). 
membre de l'Association patriotique de la littérature du Japon (Nihon bungaku hōkoku-kai 日本文学報国会) ne pouvait échapper, du moins formellement, à la question de sa responsabilité de guerre, alors la frontière tracée entre les opposants et les spectateurs passifs devenait pour le moins floue (Yuchi 2007 : 176-179). À ce titre, la SLNJ et son comité central étaient-ils réellement mieux placés que leurs collègues pour incarner le degré zéro de la responsabilité ? En outre, comme le rappelle Karatani Kōjin, l'autocritique à laquelle entendaient s'être livrés les communistes de la SLNJ consista, dans les faits, moins en un bilan de leur conduite pendant la guerre qu'en un simple retour au parti (Karatani 2003 [2000] : 166-169). Or, pour revenir, il fallait bien d'une manière ou d'une autre s'en être éloigné un moment. Et si, en effet, écart il y avait eu, quelle en était la nature exacte, et quel type de responsabilité était-il susceptible d'impliquer?

\section{La responsabilité d'après-guerre des écrivains}

En novembre 1955, soit presque une année avant que Maruyama ne s'exprime publiquement sur la question, Yoshimoto abordait dans un article intitulé "Les poètes de la génération précédente " le même problème, quoique de manière nettement plus tranchée :

Le poison distillé par la révolution pacifique ne tient pas à l'idée d'accomplir pacifiquement une révolution dans un état de servitude préalable (si tant est qu'une si belle chose soit possible). Il réside plutôt dans le fait que, à se masturber dans l'illusion d'avoir mis un terme à la guerre par leurs propres moyens, quand bien même la défaite fut le fait des troupes alliées, les forces démocratiques ont permis les mensonges dans leurs propres rangs pour, perchés sur leur piédestal, mieux cracher sur les autres camps, et s'attirer ainsi la méfiance du peuple [jinmin 人民]. (Yoshimoto 2014a : 265)

Nous pouvons observer ici, outre son ton virulent, quelques-unes des caractéristiques du second débat sur la responsabilité de guerre des écrivains ${ }^{23}$. En premier lieu, Yoshimoto et Takei ancrent leur démarche dans le présent ;

23. Afin de ne pas multiplier vainement les références à des auteurs et critiques littéraires très peu connus sous nos latitudes, nous restreindrons nos analyses aux textes suivants : "Les poètes de la génération précédente ", "La responsabilité de guerre d'après-guerre et la littérature démocratique " et "Critique de la "littérature démocratique" ". 
autrement dit, à leurs yeux, c'est la situation actuelle qui exige un réexamen du passé. Tous deux partagent alors un même sentiment d'échec vis-à-vis de la "révolution démocratique ", tant sur les plans politique que littéraire. Pour reprendre les termes de Yoshimoto, le "poison" des tenants de la "révolution pacifique" ne se trouve pas dans le fait que d'aucuns aient pu croire à leurs stratégies, mais bel et bien dans le fait qu'ils se soient concrètement aliénés les masses pour des chimères. Dans cette perspective, si le présent représente certes le résultat d'actions et d'erreurs passées, il constitue avant tout pour les analyses de Takei et Yoshimoto à la fois un point de départ et un enjeu. En ce sens, leurs travaux pourraient s'apparenter à une tentative d'archéologie critique du présent qui, plus qu'à rétablir une vérité historique dissimulée, chercherait à actualiser le passé.

Ensuite, même si les propos de Yoshimoto ci-dessus ne permettent pas, seuls, d'appuyer suffisamment ce point, cette deuxième polémique prend pour cible principale la SLNJ. Les travaux de Yoshimoto et Takei s'attachent ainsi à remettre en cause le mythe de l'innocence sur lequel elle s'était constituée, et au nom duquel elle renvoyait les " autres camps " face à leurs responsabilités. Car, parmi ses membres fondateurs, certains avaient aussi, pendant la guerre, rédigé des textes compromettants.

Tel était, par exemple, le cas du poète Tsuboi Shigeji, à propos duquel Takei écrit en mars 1956 dans «La responsabilité de guerre d'après-guerre et la littérature démocratique »: " Je ne cherche pas à dénoncer la responsabilité de guerre de Tsuboi. J'interroge sa responsabilité d'après-guerre qui commence par l'esquive de sa responsabilité de guerre " (Takei 1960 : 120). Sans trop entrer dans les détails, il n'est peut-être pas inutile de rappeler le contexte de l'affaire. Les éditions Aoki avaient publié en 1954 trois volumes de poèmes de Tsuboi, postfacés par Odagiri Hideo. Ces recueils ne prétendaient certes pas rassembler les œuvres complètes du poète ; toutefois, certains " oublis ", notamment quelques poèmes de guerre, pouvaient paraître dérangeants, voire suspects, au regard de l'attitude intransigeante de la SLNJ. La postface d'Odagiri s'abstenait également de mentionner ces poèmes, préférant se concentrer sur l'éloge de Tsuboi, dont il célébrait le talent et l'intégrité. Dans "Les poètes de la génération précédente ", Yoshimoto avait lui aussi traité de ce cas. Il y mettait côte à côte deux poèmes de Tsuboi. L'un, écrit pendant la guerre, chantait les adieux déchirés du poète à sa théière, qui s'apprêtait à aller nourrir l'acier des canons ; 
l'autre, rédigé après-guerre, mettait en scène une réunion familiale chaleureuse autour d'une même théière. Yoshimoto commentait :

Comme il était censé avoir donné durant la guerre sa théière à la ferraille, peutêtre en a-t-il acheté une nouvelle, ou peut-être s'est-il contenté d'écrire un poème dans lequel il la donnait, mais la garda pour lui. (Ô résistance plébéienne !) Ce qui m'intéresse, c'est que consciemment ou non, ces deux poèmes partent d'une même idée, et, qu'entre les deux, il y eut la guerre. À partir de là, la seule différence est que l'un s'achève sur un appel à l'agitation pseudo-fasciste, et l'autre dans une mièvrerie pseudo-démocratique. Si c'est cela être poète, les premiers sentiments que j'éprouve sont la honte, l'humiliation et le désespoir. On n'y trouve pas la moindre trace d'une lutte intérieure ni d'une accumulation sensible, indiquant quelle expérience fut subjectivement pour lui celle de la guerre. En forçant le trait, on pourrait dire que chez Tsuboi, où les questions de réorientation et de responsabilité de guerre ne se posent pas, il n'y a rien d'autre qu'une allure de plébéien, qui se contente de défiler avec son temps. (Yoshimoto 2014a : 268)

Le suivisme et l'inauthenticité reprochés ici à Tsuboi sont, pour Yoshimoto, les symptômes d'un problème plus général : celui de la subjectivité. Celle de Tsuboi appartient, selon lui, à ce qu'il nomme la " plèbe " (shomin 庶民), qu'il oppose au "peuple» (jinmin 人民). Le régime plébéien de la subjectivité n'interdit certes pas quelques modes de résistance à l'ordre, mais la capacité de ces derniers à agir sur la réalité est nulle. Il en découle que les actions d'un tel sujet ne peuvent que prendre la forme de pseudo-actions. Pour Yoshimoto, seul un travail de théorisation, procédant d'une confrontation entre le "monde intérieur » du sujet et la réalité extérieure, permet de s'émanciper de sa condition "plébéienne ". Parvenu au terme de ce processus, le sujet intérieurement théorisé se retrouve à même de comprendre et affronter l'ordre social, autrement dit de conduire une révolution ${ }^{24}$. Ainsi, selon Yoshimoto, la résistance que « le plébéien » Tsuboi entendrait opposer à la réalité par son engagement artistique, ne serait en somme guère plus qu'une illusion engendrée par l'inconscience d'une subjectivité défaillante.

24. La problématique de la subjectivité connaît à l'époque chez Yoshimoto diverses formulations. Celle présentée ici est exposée dans "Les poètes de la génération précédente ». Pour Yoshimoto, l'année 1937 dénote de manière générale l'effondrement du sujet (shutai 主体) japonais moderne qu'il observe chez Takamura Kōtarō (Yoshimoto 2014b [juillet 1955] : 102-107). 
Dès lors le silence de Tsuboi, comme celui d'Odagiri, quant à sa réorientation et ses responsabilités de guerre, n'apparaît plus comme un simple refus d'avoir à rendre des comptes, mais se double d'un aveuglement " plébéien " face à ces questions.

C'est le même type de silence et d'aveuglement que Takei dénonce chez le critique littéraire Kubokawa Tsurujiro ${ }^{25}$. Rééditions partielles de certains recueils d'essais critiques, textes de présentations articulés sur l'acuité et la constance de l'auteur : les circonstances de l'affaire étant assez similaires à celles du cas Tsuboi, nous ne nous étendrons pas dessus. Dans son réquisitoire, Takei tente, au nom précisément d'une résistance authentique de Kubokawa, de remettre en question la thèse d'une continuité dans son œuvre, autrement dit, il cherche à distinguer des périodes afin d'isoler un point de rupture. Pour Takei, ce basculement se situe au tournant des années 1940, entre l'intensification du conflit sur le continent et le début de la guerre du Pacifique. Jusqu'alors, Kubokawa avait emprunté une route parallèle aux premiers réorientés de la NALP, c'est-à-dire qu'il s'était détaché d'un réalisme dit socialiste pour se diriger vers un réalisme d'ordre plus général, affranchi de contraintes politiques. Puis, d'une conception autonome de la littérature, il serait brusquement revenu à une théorisation nettement plus politisée, au service cette fois, de la guerre et de la sphère de coprospérité asiatique.

Pour étayer son argument, Takei cite de longs passages d'un texte intitulé "La place de la littérature japonaise " (Nihon bungaku no ichi 日本文学の 位置), rédigé par Kubokawa en commémoration de la cérémonie inaugurale de l'Association patriotique de la littérature du Japon (le 18 juin 1942). Après s'être félicité de cette réunion des écrivains et des plus grands représentants de l'armée et de la marine sous la bannière de la littérature, Kubokawa se disait ému par l'allocution du Premier ministre Tōjō Hideki 東条英機 (1884-1948), enjoignant les membres de l'association à la bonne entente. Kubokawa poursuivait en affirmant qu'il était nécessaire, pour atteindre les objectifs fixés par l'association, que chaque auteur prenne conscience de sa place, mais aussi de la situation et du rôle historiques qu'étaient appelées

25. La présentation la plus détaillée et aboutie qu’il consacre à Kubokawa se trouve dans "La responsabilité de guerre d'après-guerre et la littérature démocratique " (Takei 1960 [mars 1956] : 132-141). 
à tenir les Lettres japonaises au sein de la littérature mondiale. Or cette vision du monde, ajoutait-il, était indissociable de la politique culturelle nationale, dans la mesure où elle ne pouvait lui être que subordonnée. Sa réalisation exigeait donc que les écrivains, ceux notamment qui concevaient l'art comme séparé de la politique, procèdent à leur autocritique littéraire. Takei commente :

Ne voyons-nous pas ici une minable caricature de théorie littéraire marxiste ? À l'instar d'un grand nombre d'ex-marxistes qui, pendant la guerre, mirent leurs théories des forces productives et leurs discours sur l'autodétermination des peuples au service d'une rationalisation de l'économie de guerre et de l'établissement de la sphère de coprospérité de la grande Asie, Kubokawa Tsurujirō dévoile ici, comme théoricien à la solde de la littérature impériale, les résidus de son passé de marxiste. (Takei 1960 [mars 1956] : 139).

Toutefois, selon Takei, la présence de Kubokawa, suite à son repositionnement démocratique après la défaite, parmi les membres fondateurs de la SLNJ, dont seuls étaient censés être dignes " ceux qui n’ont pas collaboré à cette guerre impérialiste et s'y sont opposés ", interdisait de fait un retour sur son activité réelle, c'est-à-dire sa réorientation, pendant le conflit ${ }^{26}$.

Le problème de la réorientation occupe une place centrale dans les articles de Takei et de Yoshimoto. Cela tient probablement au fait qu'il est alors largement envisagé à travers le prisme d'une non-réorientation, conçue à la fois comme forme de fidélité au communisme, mais aussi, et par là-même, comme une sorte d'échappatoire à la question des responsabilités de guerre. Les procédures mises en œuvre par Yoshimoto et Takei visent surtout à troubler cette configuration. Cela ne signifie pas qu'ils s'en détournent, ni qu'ils la rejettent catégoriquement, mais plutôt qu'ils l'exploitent pour interpréter un silence.

Tous deux n'ignorent pas que la réorientation des membres de la NALP incarcérés au début des années 1930 a été le fruit de pratiques coercitives

26. Selon le poète anarchiste Akiyama Kiyoshi 秋山清 (1904-1988), les propos de Takei furent très mal accueillis, particulièrement par Miyamoto Kenji et Kubokawa lui-même, lors de l'assemblée des cadres de la SLNJ de juin 1956. Akiyama, pour sa part, déplora l'attitude de ses collègues et considérait que le sujet devait être ouvertement débattu avec la jeune génération (Akiyama 2006 [1956] : 180-184). 
particulièrement brutales, ni que la police tortura à mort Kobayashi Takiji 小林多喜二 $(1903-1933)^{27}$. Ils n'ignorent pas, non plus, que le terme évoque aussi le reniement de Sano Manabu 佐野学 (1892-1953) et Nabeyama Sadachika 鍋山貞親 (1901-1979), et leur fameuse « Déclaration à nos camarades conjointement accusés " (Kyōdō hikoku dōshi ni tsuguru sho 共同被告同志に告ぐる書), exhortant à la réorientation, et les répercussions qu'on lui prête ${ }^{28}$. Takei et Yoshimoto ont donc une certaine connaissance du contexte historique de l'effondrement du mouvement prolétarien, qui influence sans doute en partie le regard qu'ils portent sur l'acte de réorientation. C'est du moins en contraste avec les réorientations du début des années 1930 qu'ils entendent rendre compte de la spécificité de celles des années 1940. La définition la plus concise de ce qu’ils nomment, parodiant en cela la formule révolutionnaire des thèses de 1932, " la réorientation en deux stades» (nidankai tenkō-ron二段階転向論) se trouve dans "Critique de la "littérature démocratique" ». Yoshimoto y écrit :

Dans mon esprit (et bien que je n'aie pas encore assez de documents à disposition), l'échec du mouvement littéraire prolétarien se divise en deux stades, articulés autour des années 1937-1940.

À la première période correspond un processus au cours duquel, fauchés à la racine des défaillances historiques de leur mouvement par la répression, certains se retrouvèrent isolés, battirent en retraite et se réorientèrent ; à la seconde, un processus au cours duquel, usant des aptitudes et théories jadis acquises durant l'âge d'or de la

27. Pour une description des conditions de détention, voir Mitchell (1976: 109-147), et Steinhoff (1991: 100-103).

28. La publication de ce texte en version abrégée, en juin 1933 dans le Asahi shinbun 朝日新聞, suivie en juillet de sa version intégrale dans la revue Kaizo 0 改造 (Réforme), est souvent considérée comme le déclencheur de la première grande vague de réorientations, parfois qualifiée de "réorientations de masse » (tairyō tenkō 大量転向). Il semble en effet que les déclarations officielles pour les prévenus en attente de jugement s'élevaient alors à près de $30 \%$, atteignant même $34 \%$ en ce qui concerne ceux jugés le mois suivant la diffusion de la fameuse déclaration. Fauconnier considère cependant que l'influence du texte de Sano et Nabeyama doit être relativisée. Si la parution a bien été suivie d'un nombre conséquent de réorientations, le phénomène n'est pas aussi massif que son qualificatif le laisse supposer : les prévenus étaient certes pour beaucoup classés à gauche de l'échiquier politique, mais leur nombre ne permet pas de parler de "vague générale " de conversions (Fauconnier 2012 : 263-264 et 295-296). 
littérature prolétarienne, certains se rangèrent du côté de l'autorité, et se livrèrent à une réorientation active en rationalisant sa politique artistique.

Les premières réorientations, dont l'emblème demeure le meurtre atroce que perpétra l'autoritarisme sur la personne de Kobayashi Takiji, ont l'oppression pour cause principale; quant aux secondes, que l'on ne peut attribuer à l'oppression, elles sont en quelque sorte la volte-face (jiko kaiten 自己回転) des défaillances théoriques, pratiques et organisationnelles du mouvement littéraire prolétarien pour se reproduire. Il en résulte que les premières réorientations se firent "contre leur gré " et les secondes de "plein gré ». (Yoshimoto 2014a [avril 1956] : 289)

Le caractère approximatif des diverses dates avancées par Yoshimoto et Takei pour tenter de situer la deuxième phase de ces réorientations est, à notre avis, secondaire, dans la mesure où la distinction qu'ils cherchent à établir est visiblement de nature plus conceptuelle, ou métaphorique, que chronologique $^{29}$. Plus qu'une opposition entre époques, il s'agit de produire un contraste entre plusieurs types d'attitudes subjectives. Il faut sans doute rappeler que, dans ce schéma, les premiers réorientés ${ }^{30}$ sont, avant d'être comparés aux seconds, opposés aux premiers non-réorientés ${ }^{31}$. Selon Yoshimoto, en effet, l'alternative qui leur était laissée consistait à renier leur

29. On peut par ailleurs supposer que ces imprécisions sont la marque d'une connaissance issue de leur expérience personnelle d'adolescent, ou du souvenir qu'ils en conservent, plus que d'une culture à proprement parler livresque.

30. Yoshimoto fait ici explicitement référence à Nakano Shigeharu et Miyamoto Yuriko. Figure majeure de la littérature prolétarienne d'avant-guerre, Nakano fut arrêté en 1932 pour appartenance au PCJ. Il se réorienta en 1934, c'est-à-dire qu'il officialisa auprès des autorités son renoncement au communisme. À sa libération, il se lança dans l'écriture de récits à caractère parfois ouvertement autobiographique, à travers lesquels il narrait ses expériences de réclusion, de peur, de désenchantement et d'échec, devenant ainsi l'un des premiers et plus éminents représentants de ce qui, à l'époque déjà, fut nommé la "littérature réorientée » (tenkō bungaku 転向文学). Après-guerre, il réintégrera le PCJ, qu'il représenta à la Chambre Haute de 1947 à 1950. Dans le cas de Miyamoto Yuriko, la "réorientation " fut moins nette que ne le laisse entendre Yoshimoto. En effet, en dépit de multiples arrestations et d'une santé fragile, elle partagea son activité en temps de guerre entre la littérature et le soutien à son mari incarcéré, Miyamoto Kenji. Yoshimoto semble ici surtout faire allusion à son court essai "Les bourgeons qui survivent à l'hiver " (Fuyu wo kosu tsubomi 冬を越す蕾, 1934) dans lequel elle invoquait la nécessité d'une retraite stratégique, c'est-à-dire une suspension temporaire des objectifs littéraires et politiques du mouvement prolétarien, afin de continuer à produire des œuvres.

31. Yoshimoto mentionne les noms de Miyamoto Kenji et Kurahara Korehito. 
mouvement afin de poursuivre leurs activités sous une forme modifiée, ou à les garder inchangées et les suspendre, à l'image des non-réorientés. Il s'agit en somme d'un non-choix, d'un choix subi qui, dans un cas comme dans l'autre, débouche pour Yoshimoto sur la disparition de la pensée et du discours marxiste dans l'espace public japonais au cours des années 1930. Le caractère binaire de ce schéma exclut donc d'emblée une troisième voie, celle d'une non-réorientation nuancée, ou d'une réorientation simulée, dont se réclameront notamment après-guerre Tsuboi et Kubokawa, invoquant eux aussi des limitations de choix imposées par les pressions du climat de guerre ${ }^{32}$.

C'est précisément le recours à cet argument que vient saper la rhétorique de Yoshimoto et Takei, en isolant le deuxième stade du premier dont les circonstances carcérales sont soulignées. Leur liberté n'étant pas en jeu, les réorientés de la deuxième vague auraient ainsi «librement " choisi de se ranger du côté des autorités. C'est du moins ce que pourrait laisser entendre l'expression de "réorientation active ". Le deuxième stade de la réorientation implique dès lors un degré de responsabilité considérablement plus élevé que le premier. De plus, et à la différence des premiers réorientés, les seconds se voient également accusés d'avoir mis leurs compétences au service de la rationalisation des politiques étatiques. Yoshimoto semble ainsi sousentendre que c'est en qualité d'ex-marxistes qu'ils auraient contribué à l'effort de guerre. La résurgence de conceptions littéraires associées à la NALP dans les années 1940 pour Kubokawa, l'attachement à certains tropes poétiques pour Tsuboi dans l'après-guerre, autrement dit une certaine forme de constance dans le langage et les attitudes, trahiraient ainsi l'inconstance de leurs convictions comme l'ambivalence de leurs engagements.

On peut alors se poser la question : pourquoi, étant donné les différences fondamentales qui séparent le premier du second stade de la réorientation, conserver le même terme pour désigner les deux ? Le point commun entre premiers et seconds réorientés, c'est que tous deux sont pensés en opposition avec les non-réorientés. Lorsque Yoshimoto et Takei qualifient ceux à qui ils reprochent de ne pas assumer leurs responsabilités d'aprèsguerre de "réorientés ", c'est principalement pour les connotations particulièrement négatives associées alors au terme. Autrement dit, les traiter de

32. C'est du moins ce que rapporte Akiyama (Akiyama 2006 [1956] : 183). 
" réorientés ", c'est avant tout leur refuser les privilèges du non-réorienté : le courage et l'innocence de la victime et la vertu du vainqueur. La typologie de Yoshimoto laisse néanmoins une question en suspens : si les " faux " non-réorientés ne sont plus à l'abri de la "réorientation ", les "véritables " non-réorientés le sont-ils toujours? C'est en décembre 1958, dans les colonnes de Gendai hyōron, qu'il y apportera une réponse, en renversant le rapport jusqu'alors établi entre réorientés et non réorientés dans un texte sobrement intitulé : «La réorientation ».

\section{Réinterpréter la réorientation}

Le mitan des années 1950 est marqué par la parution de nombreux essais d'interprétation générale sur la réorientation (tenkō-ron 転向論), qui envisagent de la redéfinir sous un angle nouveau, en tentant notamment de la désolidariser de la question des responsabilités de guerre ${ }^{33}$. Ce contexte de ré-investigation influença non seulement le texte que Yoshimoto consacre à la question mais aussi, dans une certaine mesure, sa réception.

De nos jours, la plus emblématique de ces tentatives reste probablement celle du Groupe d'étude sur la science des idées (Shisō no kagaku kenkyūkai 思想の科学研究会, ci-après GESI) réuni autour du philosophe Tsurumi Shunsuke 鶴見俊輔 (1922-2015) ${ }^{34}$. Le pluralisme des approches, tout comme la diversité des sujets considérés permettent aux travaux du GESI d'offrir un regard à la fois précis et panoptique sur la question. La réorientation y est principalement envisagée à travers la définition qu’en

33. Ce passage s'appuie sur Tobe (2006 : 322-332), et Fauconnier (2012: 60-102).

34. Le GESI partage son nom avec la revue Science des idées (Shisō no kagaku 思想の科学) : lancée en 1946 et mensuelle dès l'année suivante, elle se poursuivra jusqu'en 1996 et comptera une centaine de membres actifs. Si le groupe ne fera pas de la "réorientation " son unique objet de recherches, son importance dans l'étude de celle-ci demeure majeure. Les premières études publiées dans la revue du groupe remontent à 1953. Ces travaux donneront naissance aux trois imposants volumes Études collectives sur la réorientation (Kyōdökenkyū : tenkō 共同研究 転向), dont les deux premiers tomes paraîtront en 1959, et le troisième en 1962. Ces volumes sont respectivement consacrés à l'avantguerre, à la période de guerre et enfin à l'après-guerre. L'article que Tsurumi consacre à Yoshimoto se trouve dans le troisième volume (Tsurumi 1978b [1962] : 328-343). 
donne Tsurumi en introduction : " un changement des idées survenu sous la coercition du pouvoir ${ }^{35}$ "(Tsurumi 1978a [1959] : 6). Les premiers chapitres du volume 1 sont par ailleurs consacrés à des analyses linguistiques et étymologiques du terme qui, aujourd'hui encore, semblent acceptées $^{36}$. La question de la réorientation ne se retrouve donc plus limitée au contexte des seules années 1930 , et la notion peut dès lors être appliquée à des populations ou individus n'ayant eu aucun lien avec le communisme. L'atténuation de ces connotations négatives reflète en partie, comme le souligne Tobe Hideaki, la nature profondément éthique de leur démarche (Tobe 2006 : 327-328).

Cette même caractéristique était déjà présente chez le critique littéraire Honda Shūgo 本多秋五 (1908-2001), lorsqu'il publia La littérature réorientée (Tenkō bungaku-ron 転向文学論) en février 1954. Dans ce texte majeur, Honda abordait la question, dont il tentait de donner une définition intrinsèquement plus littéraire que son habituelle réduction aux textes produits par des auteurs s'étant réorientés pour mettre fin à leur incarcération. Ce faisant, il caractérisait la réorientation littéraire comme un retour à des formes passées, étendant ainsi la portée de cette catégorie bien au-delà du mouvement littéraire prolétarien. Honda y soutenait aussi que la répression en avait été la cause principale (Honda 1954 : 266-274). Ainsi, dans la mesure où il referme avec obstination la problématique sur le mouvement prolétarien et ses héritiers, comme par son effort insistant de penser la réorientation en dépit du facteur coercitif, le texte de Yoshimoto peut être lu comme un démenti des thèses de Honda.

35. Nous empruntons cette traduction à Fauconnier (2012 : 133).

36. Dans sa contribution, le disciple de Maruyama, Fujita Shōzō 藤田省三 (19272003), fait ainsi remonter l'origine de la notion aux débats entre marxistes qui, dans les années 1920, opposèrent Yamakawa Hitoshi 山川均 (1880-1958) et Fukumoto Kazuo 福本和夫 (1894-1983), quant à la nouvelle ligne qu'il fallait donner au mouvement. Fujita montre qu'il s'agissait alors, dans l'espace discursif marxiste, d'une notion capitale et positivement connotée jusqu'à sa récupération et son renversement par les autorités, laissant ainsi entendre que ce ne fut sans doute pas par hasard si les réorientations des années 1930 portent ce nom (Tsurumi 1978a [1959] : 32-57). Dans «L'épineuse question du sujet dans la pensée philosophique du Japon moderne ", pour ne citer que notre source la plus récente, Asari Makoto souligne l'importance de ce rappel historique (Asari 2014: 144-145). 


\section{La réorientation selon Yoshimoto}

«La réorientation » s'articule sur deux axes qui, sans cesse, se croisent dans un mouvement dialectique complexe, pour ne se rencontrer véritablement qu'à la fin. D'une part, Yoshimoto cherche à y exposer une vision "personnelle" de la société japonaise dans son ensemble. D'autre part, il procède à l'analyse de certains types de réactions subjectives de l'intelligentsia du début des années 1930 face aux réalités de son temps. Ces deux axes se soutiennent donc mutuellement, sans qu'il ne soit jamais véritablement possible de déduire catégoriquement l'un de l'autre. En effet, si selon Yoshimoto, "sur un plan analytique, cette société se conçoit comme une combinaison de facteurs modernes et féodaux, dans l'esprit de ceux qui y vivent et y agissent, elle se manifeste sous les traits d'une concurrence complexe de facteurs, n'ayant ni début, ni fin " (Yoshimoto 2014b : 370). Autrement dit, la conscience des individus peine à en saisir la nature exacte, et c'est à l'inverse cette même conscience qui, par ses ratés, permet d'en appréhender la réalité analytique. Le caractère duel de cette réalité et la conscience fracturée des êtres qui la peuplent se trouvent donc, selon Yoshimoto, dans un rapport incongru de déduction mutuelle.

Notons par ailleurs que pour Yoshimoto, si le conflit entre facteurs modernes et féodaux n'est absolument pas propre au Japon, c'est toutefois sous une forme spécifique qu'il s'y exprime ${ }^{37}$. Dans cette perspective, la réorientation désigne " un changement intellectuel survenu dans l'intelligentsia, en raison de son incapacité à saisir les structures de la société moderne japonaise comme vision totale » (Yoshimoto 2014b [décembre 1958] : 369). Cette définition a pour corollaire que la réorientation ne se résume pas à ses causes externes, l'oppression politique, mais peut être pensée comme produit d'une action subjective. Telle est la toile de fond sur laquelle évoluent les trois figures que Yoshimoto convoque pour interpréter la réorientation.

37. Pour Yuchi Asao, la conception binaire d'une société japonaise divisée entre éléments "modernes » et " prémodernes » est chez Yoshimoto héritée des thèses de 1932 (Yuchi 2012 [1995] : 163-164). Quelle qu'en soit l'origine, nous pensons qu'elle reflète en réalité, comme le suggère Niikura Takahito, une représentation de la situation du Japon plus largement partagée, qu'il s'agisse des sciences sociales, du secteur économique ou de certaines politiques d'État jusqu’à la fin des années 1950 (Niikura 2015 : 132-133). 
Yoshimoto s'attache tout d'abord au cas de Sano et Nabeyama. Lorsqu'ils furent arrêtés en 1929, ils étaient des membres importants du comité central du PCJ. Condamnés en 1932, ils rédigèrent l'année suivante leur fameuse "Déclaration ", qu’ils adressaient de manière générale à leurs camarades déjà emprisonnés ou encore en liberté, pour les exhorter à rompre avec les diktats du Komintern et se rallier à la cause impériale. Le texte fut largement diffusé dans la presse ${ }^{38}$. Ils y affirmaient notamment que « le principe politique du Komintern qui oppose peuples (minzoku 民族) et classes, est une abstraction particulièrement inadéquate au Japon, dont la particularité sociale réside dans sa forte unité nationale (minzokuteki 民族的). " (Sano \& Nabeyama 1933 : 194) ${ }^{39}$. Leur rejet des directives du Komintern, rendues coupables, entre autres, d'avoir par leur obstination à abattre le système impérial éloigné le PCJ des masses, s'accompagne d'observations relatives à la particularité du Japon, dont l'empereur constituerait le "centre de l'unité nationale (minzokuteki)» (ibid.: 196). Ces adieux au prolétariat furent, sans surprise, perçus et dénoncés comme une trahison par le parti (Fauconnier $2012: 256$ ).

Dans les années 1950, la "Déclaration" de Sano et Nabeyama est encore considérée, par Honda notamment, comme l'élément déclencheur de la vague de réorientations de 1933 (Honda 1954 : 240-242). Ce n'est toutefois pas ce caractère " originel " qui retient l'attention de Yoshimoto. Il rejette, par ailleurs, fermement toutes ses interprétations en termes de " trahison ». Notons à ce propos qu'en 1958, le rejet de l'URSS de Staline ne dégage plus le même parfum de scandale qu'avant l'intervention soviétique en Hongrie et les révélations du rapport Khrouchtchev. Aux yeux de Yoshimoto, la « Déclaration » de Sano et Nabeyama constitue l'expression d'une pensée articulée, dont l'analyse permet de saisir une vision subjective. Quel qu'ait pu être le degré d'implication des autorités carcérales dans le texte, Yoshimoto rappelle que les deux hommes ne se rétractèrent ni à leur

38. Voir note 27. Notons que les autorités carcérales furent vraisemblablement « conciliantes ", puisqu'elles permirent aux deux hommes d'échanger directement durant les six mois que nécessita la rédaction du texte, pour finalement s'impliquer dans la publication (Steinhoff 1991: 11, Fauconnier 2012: 212-271)

39. Compte tenu du caractère très "libre ", si ce n'est parfois tronqué, des citations de Yoshimoto dans "La réorientation ", nous nous référons de préférence, pour les extraits cités, aux textes originaux. 
sortie de prison (respectivement en 1943 et 1940) ni après la guerre. Or, l'un des arguments fréquemment invoqués par Sano et Nabeyama souligne que par sa soumission au Komintern, le PCJ se serait aliéné les masses japonaises. Pour Yoshimoto, cette aliénation des masses induisit chez eux un sentiment d'isolement, dont la réorientation constitue le symptôme.

Le cas de Sano et Nabeyama acquiert ainsi une dimension paradigmatique, dans la mesure où il permet à Yoshimoto d'étendre le motif de l'isolement à l'ensemble de l'intelligentsia. Il faut toutefois préciser que seul ce sentiment d'isolement se voit attribuer ici une valeur archétypale. Autrement dit, s'il devient, selon Yoshimoto, le principal moteur de toute réorientation, il n'en détermine ni la forme ni la direction. Celle opérée par Sano et Nabeyama débouche, par exemple, sur une " capitulation inconditionnelle face aux facteurs dominants du féodalisme japonais " (Yoshimoto $2014 \mathrm{~b}$ : 376), qu'accompagne un rejet catégorique des conceptions modernistes, ou progressistes, qu'ils prétendaient défendre jusqu'alors. Notons que pour Yoshimoto, le "féodalisme japonais » renvoie entre autres au système impérial ainsi qu'au patriarcat qui, à ses yeux, ne constituent pas les résidus d'une modernité incomplète, mais bel et bien des "facteurs dominants " de l'ordre social, que le progressisme méconnaît autant qu'il les mésestime. Selon Yoshimoto, ce type de réorientation, au cours de laquelle l'intellectuel sacrifie ses convictions au maintien de son statut, pour rejoindre le cortège des masses appelant à la guerre ne présente rien de singulier, et s'applique à tous les membres de l'intelligentsia qui suivirent le chemin emprunté par Sano et Nabeyama.

Si la figure de la réorientation esquissée ci-dessus peut être caractérisée sommairement par un renoncement de l'intellectuel à son rôle, celle dont il sera maintenant question pourrait être présentée comme son double inversé, dont la caractéristique principale serait l'entêtement. En effet, dès lors que le sentiment d'isolement ne conditionne pas le type de réaction de l'intelligentsia, cette dernière se retrouve libre de préférer sa propre mise en quarantaine à une réintégration au courant des masses. Yoshimoto donne à cette forme de réaction intellectuelle le curieux nom de " modernismus japonais " (ibid. : 381). Parmi les trois figures qu'il mobilise dans "La réorientation ", seule celle du modernisme japonais a pour particularité de n'être pas directement associée à une personne. Une lecture attentive suggère toutefois que ce terme renvoie avant tout aux marxistes non-réorientés. En effet, les seuls noms que Yoshimoto mentionne à propos du modernisme 
japonais sont ceux de Kobayashi Takiji, Kurahara Korehito et Miyamoto Kenji. C'est probablement ce dernier, alors Premier secrétaire du PCJ, qu'il vise en particulier. Selon Yoshimoto, face à la réalité de la société japonaise, le moderniste se replie dans ses idées : pour lui "la pensée elle-même n’a jamais à répondre des structures réelles de la société ; elle se complète ellemême par l'automatisme de sa propre logique " (ibid.: 381). L’idéalisme du moderniste japonais le condamne donc à ignorer la réalité. En ce sens, le « modernisme japonais " n'éprouve jamais le besoin de se réorienter. La figure du non-réorienté reste donc prisonnière du concept de réorientation tel que Yoshimoto le définit, dans la mesure où c'est sa non-réorientation qui, précisément, l'empêche de rencontrer la réalitéto.

Ce renversement de perspective est considérable. En effet, nous l'avons vu, la figure du non-réorienté occupait une place privilégiée dans l'ordre du discours et des représentations. Yoshimoto inverse ici, de manière extrêmement provocatrice, la pyramide construite jusqualors, pour faire du nonréorienté celui qui, refusant de voir le réel en face, en devient incapable de toute remise en question. Nous avons déjà observé, avec le schéma des deux stades de la réorientation, des procédures analogues de retournement du sens à partir d'un même élément. Dans le cas présent, Yoshimoto opère le même type de transposition : c'est le courage même, prêté d'ordinaire au non-réorienté, qui devient ici le signe de son mépris, ou de son indifférence pour la société réelle. Replacé dans le contexte du Japon de 1958, dont les milieux progressistes étaient hantés par la crainte d'un retour des années trente symbolisé par l'accession de Kishi Nobusuke 岸信介 (1896-1987) au poste de Premier ministre, ce portrait du «non-réorienté réorienté »

40. On peut imaginer que ce type de représentation du non-réorienté trouve en fait, de manière rétrospective, son origine dans l'après-guerre. Oguma Eiji rappelle, en effet, que les comportements autoritaires de Tokuda ou Miyamoto Kenji étaient probablement en partie dus à une certaine méconnaissance de la situation et du quotidien de leurs concitoyens pendant la guerre (Oguma 2002 : 185). Yuchi Asao propose quant à lui de renverser le problème, et émet l'hypothèse que, dans " La réorientation ", l'isolement de l'intelligentsia constituerait en fait le contrepoint nécessaire à la thématique de l'engouement présumé des masses pour la guerre suite à l'incident de Mukden, motif cher, note-t-il, à toute une littérature vulgarisée qui vit le jour au cours des années 1930. Pour appuyer sa thèse, il met en parallèle le texte de Yoshimoto avec quelques ouvrages d'histoire de la littérature, du cinéma et du théâtre nationaux abondamment lus dans les années 1940 (Yuchi 2012 [1995] : 159-160). 
pouvait résonner comme un sombre présage, ainsi que comme une critique acerbe adressée à la gauche institutionnelle.

Entre le renoncement et l'entêtement, Yoshimoto entrevoit toutefois la possibilité, certes fragile et éphémère, d'une résolution dialectique au problème de la réorientation dans l'œuvre de Nakano Shigeharu. Plus précisément, c'est à travers la figure de Benji, personnage principal de La maison au village (Mura no ie 村の家), qu'il semble par ailleurs considérer comme un alter ego de l'auteur, que Yoshimoto conçoit la scène d'une authentique rencontre entre consciences populaire et intellectuelle. Dans cette nouvelle publiée en 1935, soit une année après sa réorientation, Nakano narre l'histoire de ce fils d'un petit fonctionnaire de province qui quitte sa campagne natale pour entreprendre des études universitaires à Tokyo. Passionné de littérature, il rejoint des cercles progressistes et intègre le parti. Son activisme politique conduit à son arrestation. Incarcéré pendant deux ans, il tente, avec le soutien de son père, de résister aux pressions policières, mais rongé par la peur, se résout finalement à la réorientation. Après sa libération, il rentre au village, où son père lui ordonne de renoncer à la plume pour se faire paysan. Ầ quelques nuances près, les trajectoires de Benji et Nakano semblent donc se confondre ${ }^{41}$.

Tout en lui concédant un caractère plus humain, Yoshimoto assimile dans un premier temps la réorientation de Benji/Nakano à celle de Sano et Nabeyama. Si l'identification du personnage de Benji à la personne de Nakano est à bien des égards problématique, elle peut cependant s'avérer productive si nous considérons que le couple Benji/Nakano constitue, pour Yoshimoto, une figure de la réorientation. Outre ses romans et nouvelles, Nakano contribua à véhiculer durant les années trente une représentation malheureuse, mais complexe, de la réorientation à travers ses essais. Yoshimoto s'appuie principalement sur « À propos de "À propos des écrivains” " (“Bungakusha ni tsuite” ni tsuite "文学者に就て」について), un texte publié en février 1935, lors de l'une des premières polémiques

41. Nakano naquit dans une famille de petits fonctionnaires de Fukui. Il entra à l'université impériale de Tokyo en 1924, et intégra peu après un cercle d'études sur le marxisme. Il contribua dès lors en tant que poète et romancier à l'essor du mouvement littéraire prolétarien jusqu’à son arrestation en 1932. Il se réorienta en 1934 et renoua avec la littérature. 
autour de la valeur à attribuer à la littérature réorientée. Nakano y écrivait notamment : "Dès lors que nous avons exposé nos faiblesses, c’est dans la crainte d'un retour à la vie de Kobayashi, dont la mort nous a séparé, qu'il nous faut commencer à vivre, c'est pourquoi il nous faut soutenir, en tant qu'écrivains, ceux qui l'ont tué. Le pourrons-nous ? Le fait que j'aie trahi le parti, que j'aie trahi la confiance que les masses lui portaient, ne s'effacera jamais» (Nakano 1935 : 162). Son évocation du retour spectral de Kobayashi, ainsi que le remord qu'il exprimait quant à sa trahison du parti ne manquent pas d'évoquer l'image d'un apostat en repentir. Toutefois, s'il acceptait l'idée qu'en tant que réorienté, il ne lui restait en somme guère plus qu'à vivre pour survivre, cette résignation constitue aussi le signe, pour Yoshimoto, d'un véritable retour de Nakano sur lui-même.

C'est précisément sur ce point que le parallèle élaboré par Yoshimoto entre Benji et Nakano permet une résolution dialectique de la réorientation. De retour au village après sa libération, Benji est violemment sermonné par son père. Ce dernier lui reproche tour à tour d'être rentré vivant, d'avoir sacrifié ses convictions et de les bafouer encore en poursuivant son activité d'écrivain. Benji entend et accepte les critiques de son père, mais lui fait savoir qu'il ne peut se résoudre à arrêter d'écrire. Yoshimoto commente : "Benji, de par sa capitulation, voit distinctement sous ses yeux le véritable ennemi qu'il doit affronter. Autrement dit, il transforme sa capitulation face à la dominance féodale japonaise, en une opportunité de saisir la forme véritable de ce qu'il doit affronter " (Yoshimoto 2014b : 376). Dans la perspective de Yoshimoto, il n'est sans doute pas anecdotique que cet ennemi adopte les traits d'un père, qui plus est aimant, dont la colère exprime la profonde déception des espoirs qu'il avait placés dans son fils ${ }^{42}$. L'injonction à un retour à l'ordre ne provient donc pas d'un rapport de forces de type policier, mais manifeste au contraire un régime de représentations plus diffus et plus commun. Le refus d'obtempérer de Benji témoigne ainsi, aux yeux de Yoshimoto, d'une prise de conscience de la nature erronée de

42. Dans l'analyse qu'il consacre à "La réorientation ", Suga Hidemi souligne que la figure des "masses ", c'est-à-dire celle du père tel qu'il est décrit dans La maison au village, appartient à la petite bourgeoisie provinciale. Comparant "La réorientation " à d'autres textes et poèmes de Yoshimoto, Suga montre que, contrairement à ce que la terminologie employée pourrait laisser entendre, les masses ont dans les années 1950, pour Yoshimoto, les traits de la classe moyenne (Suga 2008 : 59-66). 
l'alternative entre féodalisme et modernité qui, selon lui, doit au contraire être appréhendée au prisme de l'union des contraires.

Pour Yoshimoto, cette réorientation n'en est dès lors plus véritablement une, dans la mesure où, si la rencontre avec la société "réelle » entraîne l'abandon du statut privilégié de l'intellectuel, elle ne le conduit pas pour autant au rejet subjectif de son rôle de critique. Remarquons néanmoins que Benji/Nakano ne transforme pas pour autant sa " défaite " en victoire. Yoshimoto précise du reste en conclusion, sans fournir de détails, que cette troisième voie connaîtra l'échec pendant la guerre du Pacifique. En ce sens, cette "réorientation que l'on pourrait presque taxer d'inévitable", cette " réorientation qui n'en est pas une " (Yoshimoto 2014b : 374), n'a de valeur que relative. Elle n'apparaît qu'au prisme de la triade mise en scène par Yoshimoto. "La réorientation " ne cherche donc aucunement à lever la condamnation morale qui pèse sur les réorientés, mais se contente d'en renverser la hiérarchie.

\section{Réception et critique de Tsurumi}

Force est de constater que, de nos jours, l'argument et la conclusion de Yoshimoto peuvent laisser perplexe. Cela tient sans doute en partie au fait que le texte déçoit certaines des attentes qu'il suscite, à commencer par la promesse fanfaronne de "régler une fois pour toutes la question de la réorientation" (ibid. : 368). Les nombreuses entorses à la logique que génèrent des expressions telles que " non-réorienté réorienté », ou " réorientation qui n'en est pas une ", outre leur caractère déconcertant, contribuent immanquablement à affaiblir les ambitions analytiques du texte. En ce sens, Tsurumi a raison de pointer la confusion qu'entretiennent les divers usages de la notion de réorientation chez Yoshimoto (Tsurumi 1978b [1962] : 335). Il reconnaît cependant qu'en dépit de son manque de rigueur, le message que cherche à délivrer l'article est, à ses yeux, capital. Autrement dit, même formulée dans une langue équivoque, la critique adressée aux communistes non-réorientés était à l'époque clairement audible, et aussi, dans une certaine mesure, partagée. La sympathie dont témoigne Tsurumi pour le travail de Yoshimoto est peut-être toutefois le fruit d'un malentendu. Non pas, évidemment, que le message délivré soit en soi différent, mais plutôt parce que son sens est indissociable des usages a priori incohérents du terme réorientation. 
Tsurumi se penche en effet sur le texte de Yoshimoto dans le cadre des recherches du GESI. S'il souligne à plusieurs reprises que la réflexion de Yoshimoto s'est développée indépendamment des activités du groupe et que ses méthodes comme ses résultats en diffèrent, la lecture qu'il en donne reflète toutefois discrètement l'agenda de son propre projet sur certains points ${ }^{43}$.

Un premier élément peut être dégagé à partir du portrait que Tsurumi dresse de Yoshimoto en nihiliste. Le recours à une approche de type biographique est caractéristique de la méthode de Tsurumi. Elle lui permet de rendre compte, avec une acuité dynamique, des relations entre un sujet et son milieu. Considérant l'individu comme point de départ de sa pensée, Tsurumi est en outre amené à redéfinir constamment les catégories qu'il lui applique. En ce sens, le nihilisme de Yoshimoto, quoique comparé à celui de l'écrivain Haniya Yutaka 埴谷雄高 (1909-1997), est un nihilisme sur mesure. Dans sa brève biographie de Yoshimoto, Tsurumi évoque son parcours scolaire et ses débuts de poète. Il s'attarde davantage sur son «Essai sur l'Évangile selon Matthieu » (Machiu-sho shiron マチウ書試論), ainsi que sur Takamura Kōtarō (高村光太郎) ${ }^{44}$, avant d'aborder la polémique qui, au début des années 1960, oppose encore Yoshimoto au critique marxiste Hanada Kiyoteru 花田清輝 $(1909-1974)^{45}$.

Deux remarques s'imposent ici. Premièrement, Tsurumi semble privilégier les textes dans lesquels Yoshimoto tente d'exposer une vision personnelle du monde, ou de lui-même. Tsurumi ne prend donc pas en compte ses travaux plus circonstanciels, notamment ceux qu'il consacre à la responsabilité de guerre des écrivains, dont plusieurs sont pourtant reproduits

43. Il est à noter que Yoshimoto participa en mars 1959 à une table ronde organisée par le GESI (voir Tsurumi 1978b [1962] : 346-376), c'est-à-dire avant la parution, et probablement même la rédaction, de la version définitive du texte que Tsurumi lui consacre (ibid. : 328-343).

44. Publié à l'origine à compte d'auteur en 1954, l' "Essai sur l'Évangile selon Matthieu ", fut republié en 1957 dans le recueil Résistance et échec artistiques (Geijutsuteki teikō to zasetsu 芸術的抵抗と挫折). Quant à Takamura Kötarō, il s'agit d'une édition revue et considérablement augmentée du texte qui figurait dans La responsabilité, publié en 1957 également. On se reportera, pour plus de précisions, à la bibliographie de Tsurumi (Tsurumi 1978b [1962] : 342-343).

45. Pour une présentation détaillée du déroulement, des aspects et de certains enjeux de cette discorde, voir Suga (2008: 85-108). 
dans les ouvrages auxquels Tsurumi se réfère ${ }^{46}$. Deuxièmement, sa présentation insiste sur le caractère marginal de Yoshimoto. S'il est indéniable que l'image de Yoshimoto, ingénieur-électricien devenu poète, "homme du commun" transformé en "penseur radical " avait de quoi contraster au sein d'une élite intellectuelle qui cultivait l'entre-soi des grandes universités, force est de constater que cette image, qu'il a lui-même activement entretenue, a de nos jours intégré le poussiéreux rayon des clichés. Nous ne cherchons évidemment pas à accuser Tsurumi d'avoir succombé, ou contribué, aux charmes de la mythologie yoshimotesque, mais espérons pointer une certaine confusion entre l'originalité de Yoshimoto et son soi-disant isolement dans le paysage intellectuel. S'il n'est certes pas impossible de lire dans "La réorientation ", l'expression de la subjectivité de Yoshimoto, ainsi qu'une conception personnelle du monde qui l'entoure, une focalisation exclusive sur ces questions encourt le risque de décontextualiser sa réflexion. En effet, ce texte s'inscrit dans la séquence, bien plus courte, du débat sur les responsabilités d'après-guerre des écrivains, dont il partage les préoccupations et les codes. Cette lecture en restreint sans doute considérablement le sens. Elle permet en revanche, selon nous, d'en isoler le principe actif.

Un deuxième élément peut être dégagé à partir d'un objectif plus global du groupe d'études sur la réorientation. L'un des nombreux mérites des recherches menées par ce groupe consiste à avoir émancipé la notion pour l'appliquer à des groupes d'individus non-communistes, brisant de ce fait le monopole que le PCJ s'était attribué sur les discours relatifs à la réorientation (Fauconnier 2012 : 106 sqq.). Or, tout comme chez Honda, l'extension de la portée du terme s'accompagne d'un rejet, ou d'une tentative de neutralisation des connotations négatives qui lui sont attachées. C'est sur ce point que le texte de Yoshimoto invite au quiproquo. Avec sa définition extensive, son approche englobante et ses ambitions théoriques déclarées, "La réorientation » se donne, en apparence, toutes les caractéristiques d'un

46. C'est le cas notamment de "Critique de la "littérature démocratique" ", qui figure dans Résistance et échec artistiques, ainsi que "Les poètes de la génération précédente ", alors repris dans le recueil de 1959, Éthique du lyrisme (Jojö no rinri 抒情の倫理). 
essai d'interprétation générale ${ }^{47}$. Cependant, il nous semble qu'en y regardant de plus près, le tableau présenté apparaît sensiblement différent.

En effet, la définition de la réorientation proposée par Yoshimoto, celle d'un " changement intellectuel survenu dans l'intelligentsia, en raison de son incapacité à saisir les structures de la société moderne japonaise comme vision totale " (Yoshimoto 2014b : 369 ; nous soulignons), repose principalement sur une carence, un défaut consubstantiel à la conscience de ce qu'il nomme "l'intelligentsia». Si la notion est virtuellement susceptible d'être étendue à l'ensemble des intellectuels, Yoshimoto en restreint drastiquement le champ d'application à une intelligentsia systématiquement associée à la NALP ou au PCJ. Nous pouvons dès lors avancer l'idée que "La réorientation ", prenant en cela l'exact contre-pied des essais qui lui sont contemporains, cherche principalement à explorer, affirmer et étendre le caractère connotatif de la notion. C'est dans cette optique que prennent sens les divers usages qu'en propose Yoshimoto. En abordant de manière frontale le problème de la connotation véhiculée par le terme, la démarche de Yoshimoto se distingue donc de celle de Honda, comme de celle du GESI. En ce sens, le différend provoqué par l'approche de Yoshimoto, quand bien même celle-ci viserait de manière stratégique et déclarée ceux-là même qui ont fait de la réorientation une notion infâmante, s'étend aussi, de manière moins saillante, à ceux qui entendent l'affranchir d'un espace discursif profondément marqué par le marxisme. Son refus déraisonnable d'accorder au facteur coercitif un statut causal déterminant relève aussi, dans une certaine mesure, de la même logique. La complexité du propos de Yoshimoto résiderait ainsi dans le nœud suivant : sans être intégralement réductible à ses seuls aspects polémiques, il nous semble cependant inséparable de sa dimension circonstancielle. Autrement dit, si Yoshimoto peut penser la réorientation indépendamment de l'appareil coercitif, elle apparaît chez lui nettement moins envisageable en dehors du problème de la responsabilité d'après-guerre des écrivains.

47. Ce même point est aussi remarqué par Fuke Takahiro (2015 : 241-243). 


\section{Bibliographie}

\author{
AKIYAMA Kiyoshi 秋山清 2006 [1956] \\ Akiyama Kiyoshi chosakushū : bungaku \\ no jikohihan 秋山清著作集一文学の \\ 自己批判 (Akiyama Kiyoshi : œuvres - \\ Autocritique de la littérature), vol. 10, \\ Tokyo, Paru shuppan ぱる出版.
}

\section{ASARI Makoto 2014}

«L'épineuse question du sujet dans la pensée philosophique du Japon moderne », in Emmanuel Lozerand (dir.) Drôles d'individus. De la singularité individuelle dans le Reste-du-monde, Paris, Klincksieck : 141-149.

\section{DOWER John W. 1999}

Embracing Defeat: Japan in the Wake of World War II, New York, W.W. Norton \& Co.

\section{FAUCONNIER Brice 2007}

"Les "Red Purges" et la démocratisation du Japon, 1949-1952 », in Michael Lucken, Anne Bayard-Sakai \& Emmanuel Lozerand (dir.), Le Japon après la guerre, Arles, Philippe Picquier : 275-304.

\section{FAUCONNIER Brice 2012}

Tenkō 転向 : va-et-vient, convergences et changements idéologiques dans le Japon des années 1920-1950, Thèse de doctorat, Paris, INALCO/CEJ.

\section{FUKE Takahiro 福家崇洋 2015}

" "Sengo shisō" ni okeru tenkōron » 「戦後思想」における転向論 (Les essais d'interprétation de la réorientation dans I'« après-guerre »), in Izubara Masao 出原 正雄 (dir.), Sengo Nihon shisō to chishikijin no yakuwari 戦後日本思想と知識人の役割 (La pensée japonaise d'après-guerre et le rôle des intellectuels), Kyoto, Hōritsu bunkasha 法律文化社 : 227-252.

HONDA Shūgo 本多秋五 1954

«Tenkō bungaku » 転向文学 (La littérature réorientée), in Iwanami kōza. Bungaku 岩波講座 文学 (Cours Iwanami. Littérature), vol. 5, Tokyo, Iwanami shoten 岩波書店 : 251-294.

IWAKAMI Jun.ichi 岩上順一 1946 "Sōritsu taikai no hōkoku » 創立大会 の報告 (Rapport de l'assemblée fondatrice), Shin Nihon bungakaku-kai 新日本文学会, mars, $1: 62-65$.

KARATANI Kōjin 柄谷行人 1996 [1982] « Kenchiku e no ishi : Yoshimoto Takaaki » 建築への意志一吉本隆明 (Une volonté d'architecture : Yoshimoto Takaaki), Sai toshite no basho 差異としての 場所 (Le lieu comme différence), Tokyo, Kōdansha 講談社 : 244-257.

\section{KARATANI Kōjin 2003 [2000]}

Rinri 21 倫理2 1 (Éthique 21), Tokyo, Heibonsha 平凡社.

\section{KOSCHMANN Victor J. 1993}

« Intellectuals and Politics », in Gordon Andrew (ed.), Postwar Japan as History, Berkeley, University of California Press : 395-423.

\section{LUCKEN Michael 2013}

Les Japonais et la guerre, 1937-1952, Paris, Fayard. 
MARUYAMA Masao 丸山眞男 1995 [1956]

«Sensō sekinin-ron no mōten »

戦争責任論の盲点 (Les angles morts

de la théorie des responsabilités de

guerre), Maruyama Masao shū 丸山

眞男集 (Fuvres de Maruyama Masao),

vol. 6, Tokyo, Iwanami shoten 岩波書店：

159-164.

MITCHELL Richard H. 1976

Thought Control in Prewar Japan, Ithaca, Cornell University Press.

\section{MORTON Leith 2003}

Modern Japanese Culture. The Insider

View, Oxford, Oxford University Press.

\section{MURAKAMI Fuminobu 2005}

Postmodern, Feminist and Postcolonial

Currents in Contemporary Japanese

Culture. A Reading of Murakami Haruki, Yoshimoto Banana, Yoshimoto Takaaki and Karatani Kōjin, London \& New York, Routledge.

NAKANO Shigeharu 中野重治 1935 «"Bungakusha ni tsuite" ni tsuite» 「文学者に就て」について (À propos de 《À propos des écrivains »), Kōdō 行動, février: 153-162.

\section{NIIKURA Takahito 新倉貴仁 2015}

«Yoshimoto Takaaki » 吉本隆明 (Yoshimoto Takaaki), in Ōi Akai 大井赤亥, Ōzono Makoto 大園誠, Kagoshima Takeshi 神子島健, Wada Yū 和田悠 (dir.), Sengo shisō no saishinpan 戦後思想の 再審判 (Réévaluation de la pensée d'après-guerre), Kyoto, Hōritsu bunkasha 法律文化社 : 126-144.
ODAGIRI Hideo 小田切秀雄 1946

«Bungaku ni okeru sensō sekinin no tsuikyū » 文学における戦争責任の追求 (Poursuite de la responsabilité de guerre dans la littérature), Shin Nihon bungaku$k a i$ 新日本文学会, juin : 64-65.

OGUMA Eiji 小熊英二 2002 "Minshu » to «Aikoku ». Sengo Nihon no nashonarizumu to kōkyōsei〈民主〉 と〈愛国〉一 戦後日本のナショナリズムと 公共性 (《Souveraineté populaire» et « patriotisme ». Le nationalisme et la nature publique de l'après-guerre au Japon), Tokyo, Shinyōsha 新曜社.

\section{OLSON Lawrence 1978}

"Intellectuals and "the people": On Yoshimoto Takaaki », The Journal of Japanese Studies, 4 (2) : 327-357.

\section{SANO Manabu 佐野学}

\& NABEYAMA Sadachika 鍋山貞親 1933 « Kyōdō hikoku dōshi ni tsuguru sho » 共同被告同志に告ぐる書 (Déclaration à nos camarades conjointement accusés), Kaizō 改造, juillet : 191-199.

\section{SOEDA Kaoru 添田馨 2010}

Yoshimoto Takaaki. Ronsō kuronikuru 吉本隆明一論争クロニクル (Yoshimoto Takaaki - Chronique de ses polémiques), Tokyo, Kyōbunsha 響文社.

\section{STEINHOFF Patricia G. 1991}

Tenkō - Ideology and Societal Integration in Prewar Japan, New York \& Londres, Garland Publishing.

SUGA Hidemi 絓秀実 2008 Yoshimoto Takaaki nojidai 吉本隆明の 
時代 (L'époque de Yoshimoto Takaaki), Tokyo, Sakuhinsha 作品社.

TAKEI Teruo 武井昭夫 1960

Geijutsu undō no miraizō

芸術運動の未来像 (Portrait d'avenir des mouvements artistiques), Tokyo,

Gendai shichōsha 現代思潮社.

\section{TAKEI Teruo 2004}

Watashi no sengo. Undō kara mirai wo miru - Takei Teruo taiwashū わたしの 戦後: 運動から未来を見る一武井昭夫 対話集 (Mon après-guerre. Regarder l'avenir du point de vue des mouvements [contestataires] - Recueil de conversations avec Takei Teruo), Tokyo, Supēsu kaya スペース伽耶.

TOBE Hideaki 戸邊秀明 2006 «Tenkō-ron no senji to sengo » 転向論 の戦時と戦後 (Les essais d'interprétation sur la réorientation durant et après la guerre) in Morris-Suzuki Tessa et al. (dir.), Dōin teikōyokusan 動員·抵抗・翼賛 (Mobilisation, résistance, éloge), Tokyo, Iwanami shoten 岩波書店 : 307-334.

TOKUDA Kyūichi 徳田球一 1946 « Ippan hōkoku »一般報告 (Rapport général), Zen.ei 前衛, 1 (4) : 2-13.

TSURUMI Shunsuke 鶴見俊輔 1978a [1959]

Kyōdō kenkyū : Tenkō 共同研究: 転向 (Études collectives sur la réorientation), vol. 1, Tokyo, Heibonsha 平凡社.

TSURUMI Shunsuke 1978b [1962] Kyōdō kenkyū : Tenkō 共同研究: 転向
(Études collectives sur la réorientation), vol. 3, Tokyo, Heibonsha 平凡社.

YOSHIMOTO Takaaki 吉本隆明 1986a [1952]

"Adieux à un petit troupeau » (trad. Yves-Marie Allioux), in Anthologie de poésie japonaise contemporaine, Paris, Gallimard : 177-179.

\section{YOSHIMOTO Takaaki 1986b}

«À propos des écrivains et de leur responsabilité face à la guerre » (trad. Yatabe Kazuhiko), in Japon des avant-gardes, 1910-1970, Paris, Éditions du Centre Pompidou : 230-236.

\section{YOSHIMOTO Takaaki 1996 [1964]}

«Les fondements intellectuels de l'autonomie » (présentation et trad. Élisabeth Suetsugu), dans YvesMarie Allioux (dir.), Cent ans de pensée au Japon, vol. 2, Arles, Philippe Picquier : 183-224.

\section{YOSHIMOTO Takaaki 2011 [1958]}

« On tenkō, or ideological conversion » (trad. Wake Hisaaki), in Levy Indra (ed.), Translation in Modern Japan, New York, Routledge : 102-121.

\section{YOSHIMOTO Takaaki 2014a}

Yoshimoto Takaaki zenshū 吉本隆明 全集 (若uvres complètes de Yoshimoto Takaaki), vol. 4, Tokyo, Shōbunsha 晶文社.

\section{YOSHIMOTO Takaaki 2014b}

Yoshimoto Takaaki zenshū 吉本隆明

全集 (良uvres complètes de Yoshimoto 
Takaaki), vol. 5, Tokyo, Shōbunsha

晶文社.

YUCHI Asao 湯地朝雄 2006

Seijiteki geijutsu. Burehito, Hanada

Kyoteru, Ōnishi Kyojin, Takei Teruo 政治的

芸術 : ブレヒト 花田清輝 大西巨人 武井

昭夫 (L'art politique. Brecht, Hanada

Kyoteru, Ōnishi Kyojin, Takei Teruo),

Tokyo, Supēsu kaya スペース伽耶.

\section{YUCHI Asao 2007}

«Bungakusha no sensō sekinin : sono

konnichiteki imi » 文学者の戦争責任一

その今日的意味 (La responsabilité de

guerre des écrivains : sa signification de nos jours), Shakai hyōron 社会評論, 150 : 176-191.

\section{YUCHI Asao 2012 [1995]}

«Sairoku Taishū wa doko he itta ka :

Yoshimoto Takaaki ni okeru sengo

sekinin to sengo tenkō» 再録 大衆はど こへ行ったか一吉本隆明における戦後責任 と戦後転向 (Où sont allées les masses? Responsabilité et réorientation d'aprèsguerre chez Yoshimoto Takaaki), Shakai hyōron 社会評論, 170 : 152-184. 